\title{
Post-operative surveillance in soft tissue sarcoma: using tumor- specific recurrence patterns to direct approach
}

\author{
Mohammad Yahya Zaidi, Kenneth Cardona \\ Division of Surgical Oncology, Winship Cancer Institute, Emory University School of Medicine, Atlanta, GA, USA \\ Contributions: (I) Conception and design: All authors; (II) Administrative support: All authors; (III) Provision of study materials or patients: All \\ authors; (IV) Collection and assembly of data: All authors; (V) Data analysis and interpretation: All authors; (VI) Manuscript writing: All authors; (VII) \\ Final approval of manuscript: All authors. \\ Correspondence to: Kenneth Cardona, MD, FACS. Division of Surgical Oncology, Emory University Hospital Midtown Campus, Emory Medical \\ Office Tower, 550 Peachtree St., NE, Ste 900, Atlanta, GA, USA. Email: ken.cardona@emory.edu.
}

\begin{abstract}
Significant challenges exist in creating surveillance recommendations for soft tissue sarcomas (STS) given the vast heterogeneity of recurrence patterns between histologic subtypes. Using the most recent evidence on the natural history of STS, this review will propose surveillance strategies based on tumor location and histologic subtype.
\end{abstract}

Keywords: Recurrence; soft tissue sarcoma (STS); surveillance

Submitted Jun 06, 2018. Accepted for publication Aug 07, 2018.

doi: $10.21037 /$ cco.2018.08.03

View this article at: http://dx.doi.org/10.21037/cco.2018.08.03

\section{Introduction}

Major scientific and technological advances coupled with increased access to care have changed the perception on how various cancers exist within the population: what was once considered an acutely fatal disease in many malignancies is now viewed as a chronic condition requiring extended follow-up and surveillance. The expansion of increased access to care and screening mechanisms has led to earlier detection of malignancies which has resulted in the highest recorded prevalence of cancer survivors (1). This increase in survivorship has led to a heightened awareness of the particular issues surrounding cancer survivorship, including the challenges in coordinating long-term oncologic care (2). Although patients have benefited from the increased survival that treatment advances bring, many survivors continue to have psychosocial distress related to their status as a cancer survivor. A major cause behind these patients' anxiety is the fear of cancer recurrence (3). To ease this distress, multiple societies have crafted surveillance strategies which not only follow the natural history of each malignancy, but also are cost-effective and will not burden an already overused healthcare system (4-7). Although routine surveillance may offer several benefits including monitoring ongoing treatment, managing treatment sideeffects, providing support to patients and families, and identifying other social issues, the aim of an effective surveillance program is to detect cancer recurrence at a treatable, and potentially curable, stage (8).

\section{Surveillance in sarcoma}

Although surveillance protocols have been developed in parallel with our growing knowledge of cancer biology, many of these surveillance programs lack evidence for their effectiveness in detecting local or distant recurrences at a treatable stage. This is especially true in soft tissue sarcoma (STS), where the complex heterogeneity of the disease presents particular challenges in crafting effective surveillance strategies (9). The natural history of STS is determined largely by the histologic subtype of tumor and the anatomic site of origin. To this end, national and international groups have recommended surveillance strategies that encompass the diverse tumor behaviors that STS subtypes may exhibit. For example, the National Comprehensive Cancer Network (NCCN) (v2. 2018) has 
separate recommendations for extremity/superficial trunk STS and retroperitoneal sarcomas (RPS) (10). For patients with resected stage 1A/1B extremity/superficial trunk STS, postoperative surveillance includes a history and physical exam every 3 to 6 months for 2 to 3 years and then annually afterwards with a consideration for chest imaging, as well as baseline postoperative and periodic imaging of the primary site of disease based on the estimated risk of locoregional recurrence. For patients with resected stage 2 or $3 \mathrm{~A}$ disease, surveillance includes history and physical exam every 3 to 6 months for 2 to 3 years, then every 6 months for the next 2 years, and then annually, as well as chest imaging and baseline postoperative and periodic imaging of the primary tumor site. NCCN guidelines for surveillance for resected RPS include a physical exam with cross sectional imaging every 3 to 6 months for 2 to 3 years then every 6 months for the next 2 years and annually afterwards. These guidelines differ from other prominent society recommendations, including the European Society of Medical Oncology (ESMO) and the British Sarcoma Group, which further highlights the scarcity of evidence-based practice surrounding postoperative surveillance in STS $(11,12)$.

In an effort to better prognosticate outcomes for patients, multiple groups have developed nomograms to give a more personalized approach in predicting oncologic outcomes (13-19). These nomograms use individual clinical and pathologic characteristics to calculate prognosis based on statistical models. The benefit of using nomograms over traditional staging systems is that for rare tumors such as STS, precision and patient-centered tools can help better inform conversations between physicians and patients regarding treatment options and surveillance strategies. These nomograms however have several limitations. First, nomograms are often built on specific institutional experiences and thus they lack external validation. Second, nomograms are often constrained by the era in which they were created so as new treatments emerge in treating STS, these nomograms will have to be readjusted to maintain their applicability (20). Lastly, in informing surveillance protocols, nomograms are often limited in their efficacy in predicting location and multi-focality of tumor recurrences.

STS remains a rare malignancy even in centers specializing in cancer care, which makes crafting effective surveillance strategies difficult (21). In this review, we will discuss single- and multi-institutional studies regarding the natural history and recurrence patterns of STS and propose histology- and site-specific postoperative surveillance recommendations.

\section{Natural history and recurrence patterns in extremity and superficial truncal STS}

Of all STS, approximately $80 \%$ of tumors occur in the extremities and superficial trunk (22). There are over 75 separate histologic subtypes, each with a distinct biological behavior. Because of this diversity, studying individual tumor types can be difficult due to their rarity, and many groups have reported oncologic outcomes by grouping STS by the location of the primary tumor site, as this is a major consideration when approaching the treatment of these tumors. There are several limitations when approaching STS this way however, including challenges in effectively relaying prognostic information to patients with less common subtypes of STS. Regardless, multiple groups have published data regarding the natural history of extremity and superficial trunk STS with large cohorts of patients over several years. A recent multi-institutional study of 1,452 patients with localized extremity STS found that the 10-year overall survival (OS) for patients was $72.9 \%$ and the incidence of distant metastasis over 10 years was 25\% (15). Another study from the French Sarcoma Group which included 3,255 patients found that the incidence of death by 9 years for patients with truncal STS approached $15 \%$ and for extremity STS approached 22\%, while the incidence of local relapse in 9 years was almost $40 \%$ in patients with truncal STS and $23 \%$ in patients with extremity STS (23). This data is further supported by a smaller study of 188 patients with an extremity STS at a single-institution with a mean follow up time of 5 years which found that $13 \%$ of patients experienced local recurrence and $24 \%$ experienced a distant recurrence (24).

When considering prognostic factors which may help build surveillance programs, multiple groups have reported pathologic tumor grade as one of the most predictive factors for higher rates of recurrence. In the previously mentioned series from the French Sarcoma Group, tumor grade was an independent prognostic factor for both local and distant metastatic-free survival (23). Intermediate and high-tumor grade has been associated with a 2 to 6 -fold increase risk of local recurrence and a 5 -fold increase risk of distant metastasis $(24,25)$ When examining only those patients with high-grade extremity STS, local recurrence rates have been reported to be $12 \%$ at 5 years and $14 \%$ at 10 years (26). Histologic tumor grade however has limitations and thus should be used with caution when prognosticating a patient's future clinical course. A large study with over 1,000 patients from Japan noted that the predictive capability for survival of 
histologic grade was only applicable in three tumor subtypes: malignant fibrous histiocytoma (MFH), leiomyosarcoma (LMS), and liposarcoma (LPS) (27). This finding is further supported an additional study of 1,240 patients from the French Federation of Cancer Centers Sarcoma Group which found that histologic grade was prognostic in $\mathrm{MFH}$, unclassified sarcomas, synovial sarcomas, LMS, and LPS, but not in other STS subtypes (28). Thus, using histologic grade as the primary variable to craft surveillance programs for all 75 sarcoma subtypes should be avoided. This review will focus on the natural history of the most common histologic types in extremity and truncal STS in an effort to help build effective surveillance programs.

(I) Well-differentiated LPS (WD-LPS): patients with WD-LPS are considered to have a generally favorable overall prognosis with a 5 -year disease specific survival (DSS) of $93 \%$ (17). When these tumors recur, they almost exclusively recur locally, as distant recurrences have been rarely reported. The 5 -year local recurrence free survival (RFS) has been described to be anywhere between $86 \%$ and $98 \%$, though older studies have reported this number to be as low as $70 \%$ (29-31). It is important to note however, that WD-LPS has been associated with late ( $>5$ years) local recurrences thus suggesting the need for long-term surveillance (32).

(II) Dedifferentiated LPS (DD-LPS): recent advances in immunohistochemistry and molecular profiling have reclassified many tumors previously labeled as MFH as DD-LPS (16). For this review, clinical outcomes for MFH reported in older studies will be referenced with more recent series reporting outcomes specific for DD-LPS, though the older classification also included a more diverse tumor population which encompassed 4 to 5 different types of sarcomas thus making the ability to summarize recurrence patterns for this particular subtype especially difficult. Patients with DD-LPS have a 5 -year OS rate between $44 \%$ and $70 \%$ and a 10 -year OS rate between $38 \%$ and $43 \%(17,33-35)$. Patients with DD-LPS recur both locally and at distant sites with local recurrence rates of $22-31 \%$ and distant metastasis rates of $10-33 \%(33-36)$.

(III) Pleomorphic LPS: pleomorphic LPSs are generally aggressive tumors which frequently recur both locally and distantly. Previous groups have reported a 5-year OS rate of 40-63\%, and a 5-year DSS of $53-81 \%(37-41)$. Patients with pleomorphic sarcoma have a 10 -year local recurrence rate of up to $45 \%$ and a 10 -year distant recurrence rate of up to $50 \%$. It is important to note that for pleomorphic sarcoma, the most common site for distant recurrence is the lung (30).

(IV) Myxoid LPS: myxoid LPSs is subdivided into two groups: pure myxoid LPS and myxoid/round cell LPS. Patients with myxoid LPS generally have favorable outcomes with 5 -year DSS rates of $88-100 \%$ and 10 -year DSS rate of up to $93 \%$ $(17,29,30,37)$. Tumor recurrences are more frequently locoregional, with an overall 5 -year RFS rate of $75 \%$ and a 5 -year and 10 -year local RFS rate of $84-86 \%$ and $84 \%$, respectively; and a 5 -year and 10-year distant RFS rate of 96-100\% and $95 \%$, respectively $(15,17,29,37)$. On the other hand, patients with myxoid/round cell LPS tend to have worse outcomes in the presence of a greater than $5 \%$ round cell component placing patients at an increased risk for metastasis and death (37). For myxoid/round cell LPS, 5-year DSS rates are $74-87 \%$ and 10 -year DSS are as low as $77 \%$ $(17,37)$. Five-year and 10 -year local RFS rates are $86 \%$ and $78 \%$, respectively and 5 -year and 10 -year distant RFS are both 78\% (37). As opposed to pleomorphic LPS, the majority of distant metastases in myxoid LPS are extra-pulmonarywith abdominopelvic metastasis occurring quite frequently. The patterns of recurrence for these tumors often involve metastatic spread to the spine $(30-74 \%$ of extrapulmonary metastases), the retroperitoneum (18-71\% of extrapulmonary metastases), and the abdominal and thoracic wall (up to $9 \%$ of extrapulmonary metastases) although metastases to other sites including intraabdominally have been reported (37,42-45). Thus, surveillance strategies should include imaging these sites with appropriate cross-sectional imaging, which may include MRI.

(V) Malignant Peripheral Nerve Sheath Tumor (MPNST): MPNSTs are rare tumors which can be associated with neurofibromatosis type 1 (NF-1) or occur sporadically. The presence of a hereditary tumor syndrome has questionable association with worse prognosis as one series of 205 patients showed no difference in recurrence rates between those patients with and without NF-1, and an overall disease specific mortality of $43 \%$ at 10 years 
and disease-free survival of $40 \%$ at 10 years (46). In a separate series of 140 patients however, 10year DSS was $35 \%$ for patients with NF-1 while it was $50 \%$ for patients without NF-1 (47). Even considering these differences in survival, patients with MPNST have recurrence rates of up to $50 \%$ at 5 years which mandates strict surveillance protocols for these patients (46-48).

(VI) Myxofibrosarcoma: Although these tumors overall have a fair prognosis with 5-year OS rates between $75 \%$ and $77 \%(30,49)$, these tumors tend be locally aggressive and infiltrative and thus can have local recurrences quite often. The 5 -year local RFS rate for myxofibrosarcoma is $69-86 \%$ and the distant metastasis free survival rate is $68-83 \%(30,49-51)$. Distant metastases most commonly occur in the lung in patients with myxofibrosarcoma.

(VII) Undifferentiated pleomorphic sarcoma (UPS): as with DD-LPS, many patients with UPS were previously classified as MFH, but with current improvements in molecular profiling, more specific and recent studies for UPS are summarized in this review. Patients with UPS have been described to have a 5-year DSS of 60-63\%, 5-year local RFS rates of $73-81 \%$, and a 5 -year distant metastasisfree survival of $57-70 \%(30,52)$. Because of the high propensity of these tumors to recur both locally and distantly, surveillance imaging should focus on both the primary tumor site and the lungs in these patients.

\section{Natural history and recurrence patterns in retroperitoneal STSs}

The tumor diversity seen in extremity and superficial trunk STS, although to a lesser extent, has also been described in RPSs and, as in extremity and superficial trunk STS, these subtypes can exhibit vastly different recurrence patterns from one another. Fortunately, in the current era of sarcoma treatment, the Trans-Atlantic RPS Working Group (TARPSWG) was formed to better characterize this rare cohort of malignancies by compiling data from several high-volume sarcoma centers $(53,54)$. The TARPSWG found that for all RPS, the 5 -year local recurrence rate was $26 \%$ and 10 -year local recurrence rate was $35 \%$. This data stresses the importance of ongoing surveillance for late recurrences which may occur in RPS, an observation reported by other groups as well $(19,32)$. A few select histologic subtypes of RPS comprise the majority of tumors seen and we will focus on these:

(I) Well-Differentiated LPS: WD-LPS is the most commonly occurring histologic subtype of RPS, with an OS rate of up to $80 \%$ at 8 years $(19,53,55)$. Local recurrence rates are between $19 \%$ and $58 \%$ at 5 years and up to $60 \%$ at 15 years, with over one-third of these local recurrences being multifocal recurrences (19,55-57). Tseng et al. reported that up to $18 \%$ of these multifocal, locoregional recurrences are remote from the original resection field, in a compartment remote from the index tumor resection (58). As in the extremity, WD-LPS in the retroperitoneum has practically a $0 \%$ distant metastasis rate.

(II) Dedifferentiated LPS: as in the extremity and superficial trunk, DD-LPS has the worst overall prognosis of LPS subtypes. The 5-year OS rates range between $37 \%$ and $44 \%$, with the incidence of local recurrences being between $58 \%$ and $82 \%$ by 5 years and distant recurrences between $9 \%$ and $44 \%(19,53,55-57)$. Similar to WD-LPS, a large proportion of DD-LPS local recurrences are multifocal with up to $28 \%$ outside the original resection field, though in DD-LPS recurrences occur sooner and more frequently $(55,58)$. Grade also has prognostic importance in DD-LPS. Grade 3 tumors are the most aggressive type and have significantly worse outcomes compared to grade 2 tumors with lower 8 -year OS (30\% vs. 50\%) and higher 8 -year distant metastasis risk (30\% vs. 10\%), though local recurrence risks are similar between grades (53).

(III) LMS: LMS represents the second most common histologic subtype of retroperitoneal STS and is associated with a low local recurrence rate (6-16\% at 5 years). LMS is however associated with a high distant metastasis rate of up to $58 \%$ at 5 years $(19,53,56,57)$. These tumors also warrant extended surveillance for recurrences as they are associated with a 3 -fold higher risk for late (greater than 5 years) metastatic recurrence (32).

(IV) Solitary fibrous tumor (SFT): patients with SFT have a generally favorable prognosis given the low-malignant potential and indolent nature of these tumors. Survival at 8 years is as high as $75 \%$ and these tumors have low local recurrence rates between $4 \%$ and $8 \%$, although more aggressive 
variants of SFT do exist which tend to recur distantly at rates of up to $41 \%$ at 5 years $(19,53,56)$. A recent study from Memorial Sloan Kettering Cancer Center of 219 patients with SFTs found 5 -year and 10-year disease specific death rates of $9 \%$ and $11 \%, 5$-year and 10-year local recurrence rates of $4 \%$ and $7 \%$, and 5 -year and 10 -year distant recurrence rates of $13 \%$ and $16 \%$, though late local and distant recurrences have been reported at up to 16 years after presentation (59).

\section{Imaging modalities and recommendations for surveillance}

Presently, there is a lack of clinical trials assessing the effectiveness of varying imaging modalities and frequencies needed for optimal surveillance for patients with extremity, superficial trunk, or retroperitoneal STS. Current guidelines are informed by retrospective studies, consensus opinions, tumor biology, and cost-effectiveness models. In regard to extremity STS, common practice for surveillance includes a clinical history and physical exam to assess for local recurrences and chest radiographs to assess for pulmonary metastases, with chest computed tomography (CT) scan reserved for suspicious lesions seen on plain films (60-63). According to the NCCN guidelines, chest $\mathrm{X}$-ray can be used for chest imaging though chest CT scan without contrast is preferred. When considering cost effectiveness specifically, optimal surveillance for distant metastases for patients with intermediate to high-grade disease has been shown to be chest X-ray at longer intervals when the distant recurrence risk is less than $33 \%$ and chest CT scan when the distant recurrence risk is greater than $33 \%$ (64). Assessing for local recurrences for most patients can be done effectively with physical exam alone, as advance imaging including magnetic resonance imaging (MRI), has been shown to infrequently detect asymptomatic local recurrences $(65,66)$. When considering RPS, contrastenhanced CT scans of the abdomen and pelvis is the most appropriate imaging modality for surveillance of the abdomen, while non-contrast CT scan of the chest or plain radiographs can be considered depending on the risk of distant metastasis (67). Functional imaging with positron emission tomography (PET) scan is currently not recommended for routine use in STS surveillance due to the lack of data regarding its effectiveness (68).

Our recommendations for postoperative surveillance are informed by the primary tumor site, underlying tumor biology and clinical behavior of the STS being surveyed. For patients with more indolent extremity and superficial trunk STS (WD-LPS and pure myxoid LPS), we recommend a surveillance protocol consisting of a clinical history and physical exam with chest radiographs every 6 months for 3 years followed by annually thereafter. For patients with more intermediate- to aggressive-tumor histologic subtypes of extremity and superficial truck STS (myxoid/round cell LPS, pleomorphic LPS, DD-LPS, MPNST, myxofibrosarcoma, and UPS), we recommend clinical history and physical exam with chest CT scan without contrast every 3-6 months for 2-3 years, then every 6 months for 2 years, and then annually afterwards. For patients with myxoid/round cell LPS, obtaining a CT scan of the abdomen and pelvis with contrast at the time of obtaining chest imaging is also necessary as these tumors often recur in extra-pulmonary locations, and consideration of MRI imaging of the spine is suggested as these tumors frequently metastasize to bony areas. In regards to imaging of the primary tumor site in STS of the trunk or extremity, we would not routinely perform imaging if the site could be appropriately assessed clinically however we would consider MRI at the time of surveillance assessment for those sites that are unable to be adequately assessed clinically and/or if abnormal clinical findings were identified.

For RPS, we suggest routine postoperative surveillance for tumor recurrence, as selective surveillance initiated by patient symptoms may be too non-specific to effectively detect recurrences at a stage where intervention may be beneficial. Routine surveillance for more indolent RPS tumor types (WD-LPS and SFT) should consist of a clinical history and physical exam and cross-sectional imaging of the abdomen and pelvis (CT scan preferred or MRI) every 6 months for 3 years followed by annual assessment. For RPS tumor subtypes which are more aggressive (DD-LPS and intermediate- to high-grade LMS), surveillance should consist of clinical history and physical exam with cross sectional imaging of the chest, abdomen, and pelvis (CT scan preferred or MRI) every 4 months for 2 years followed by every 6 months for the next 3 years and then annually afterwards.

In designing surveillance regimens which more closely reflect tumor biology based on histologic subtype, we feel that the ability to detect local and distant recurrences at an interventional stage will improve for patients with STS. We also stress the importance of pairing these surveillance strategies with high-quality nomograms to better predict oncologic outcomes for patients. 


\section{Conclusions}

Unique obstacles exist in creating surveillance strategies for STS due mainly to the diverse natural history and recurrence patterns between tumor subtypes. In recent years however, multiple groups have compiled extended followup data on the specific histologic subtypes of STS. In light of this data, we propose using tumor-specific protocols for postoperative surveillance centered around the histologic subtype of each tumor.

\section{Acknowledgements}

None.

\section{Footnote}

Conflicts of Interest: The authors have no conflicts of interest to declare.

\section{References}

1. Miller KD, Siegel RL, Lin CC, et al. Cancer treatment and survivorship statistics, 2016. CA Cancer J Clin 2016;66:271-89.

2. de Moor JS, Mariotto AB, Parry C, et al. Cancer survivors in the United States: prevalence across the survivorship trajectory and implications for care. Cancer Epidemiol Biomarkers Prev 2013;22:561-70.

3. Lewis RA, Neal RD, Hendry M, et al. Patients' and healthcare professionals' views of cancer follow-up: systematic review. Br J Gen Pract 2009;59:e248-59.

4. Chen RC, Rumble RB, Jain S. Active Surveillance for the Management of Localized Prostate Cancer (Cancer Care Ontario guideline): American Society of Clinical Oncology Clinical Practice Guideline Endorsement Summary. J Oncol Pract 2016;12:267-9.

5. Khatcheressian JL, Hurley P, Bantug E, et al. Breast cancer follow-up and management after primary treatment: American Society of Clinical Oncology clinical practice guideline update. J Clin Oncol 2013;31:961-5.

6. Meyerhardt JA, Mangu PB, Flynn PJ, et al. Follow-up care, surveillance protocol, and secondary prevention measures for survivors of colorectal cancer: American Society of Clinical Oncology clinical practice guideline endorsement. J Clin Oncol 2013;31:4465-70.

7. Shirai K, Wong SL. Melanoma Surveillance Strategies: Different Approaches to a Shared Goal. Ann Surg Oncol
2018;25:583-4.

8. Rose PW, Watson E. What is the value of routine followup after diagnosis and treatment of cancer? Br J Gen Pract 2009;59:482-3.

9. Brennan MF. Follow-up is valuable and effective: true, true and unrelated? Ann Surg Oncol 2000;7:2-3.

10. Network NCC. Soft Tissue Sarcoma (Version 2. 2018). 2018. Available online: https://www.nccn.org/ professionals/physician_gls/pdf/sarcoma.pdf

11. Grimer R, Judson I, Peake D, et al. Guidelines for the management of soft tissue sarcomas. Sarcoma 2010;2010:506182.

12. Group ESESNW. Soft tissue and visceral sarcomas: ESMO Clinical Practice Guidelines for diagnosis, treatment and follow-up. Ann Oncol 2014;25 Suppl 3:iii102-12.

13. Anaya DA, Lahat G, Wang X, et al. Postoperative nomogram for survival of patients with retroperitoneal sarcoma treated with curative intent. Ann Oncol 2010;21:397-402.

14. Ardoino I, Miceli R, Berselli M, et al. Histology-specific nomogram for primary retroperitoneal soft tissue sarcoma. Cancer 2010;116:2429-36.

15. Callegaro D, Miceli R, Bonvalot S, et al. Development and external validation of two nomograms to predict overall survival and occurrence of distant metastases in adults after surgical resection of localised soft-tissue sarcomas of the extremities: a retrospective analysis. Lancet Oncol 2016;17:671-80.

16. Callegaro D, Miceli R, Mariani L, et al. Soft tissue sarcoma nomograms and their incorporation into practice. Cancer 2017;123:2802-20.

17. Dalal KM, Kattan MW, Antonescu CR, et al. Subtype specific prognostic nomogram for patients with primary liposarcoma of the retroperitoneum, extremity, or trunk. Ann Surg 2006;244:381-91.

18. Gronchi A, Miceli R, Shurell E, et al. Outcome prediction in primary resected retroperitoneal soft tissue sarcoma: histology-specific overall survival and disease-free survival nomograms built on major sarcoma center data sets. J Clin Oncol 2013;31:1649-55.

19. Tan MC, Brennan MF, Kuk D, et al. Histology-based Classification Predicts Pattern of Recurrence and Improves Risk Stratification in Primary Retroperitoneal Sarcoma. Ann Surg 2016;263:593-600.

20. Callegaro D, Miceli R, Gronchi A. Sarcoma nomograms: a light over the darkness. Oncoscience 2017;4:15-6.

21. Siegel RL, Miller KD, Jemal A. Cancer statistics, 2018. CA Cancer J Clin 2018;68:7-30. 
22. Bagaria SP, Ashman JB, Daugherty LC, et al. Compliance with National Comprehensive Cancer Network guidelines in the use of radiation therapy for extremity and superficial trunk soft tissue sarcoma in the United States. J Surg Oncol 2014;109:633-8.

23. Italiano A, Le Cesne A, Mendiboure J, et al. Prognostic factors and impact of adjuvant treatments on local and metastatic relapse of soft-tissue sarcoma patients in the competing risks setting. Cancer 2014;120:3361-9.

24. Sabolch A, Feng M, Griffith K, et al. Risk factors for local recurrence and metastasis in soft tissue sarcomas of the extremity. Am J Clin Oncol 2012;35:151-7.

25. Biau DJ, Ferguson PC, Chung P, et al. Local recurrence of localized soft tissue sarcoma: a new look at old predictors. Cancer 2012;118:5867-77.

26. Eilber FC, Rosen G, Nelson SD, et al. High-grade extremity soft tissue sarcomas: factors predictive of local recurrence and its effect on morbidity and mortality. Ann Surg 2003;237:218-26.

27. Hashimoto H, Daimaru Y, Takeshita S, et al. Prognostic significance of histologic parameters of soft tissue sarcomas. Cancer 1992;70:2816-22.

28. Coindre JM, Terrier P, Guillou L, et al. Predictive value of grade for metastasis development in the main histologic types of adult soft tissue sarcomas: a study of 1240 patients from the French Federation of Cancer Centers Sarcoma Group. Cancer 2001;91:1914-26.

29. Chang HR, Hajdu SI, Collin C, et al. The prognostic value of histologic subtypes in primary extremity liposarcoma. Cancer 1989;64:1514-20.

30. Smith HG, Memos N, Thomas JM, et al. Patterns of disease relapse in primary extremity soft-tissue sarcoma. $\mathrm{Br}$ J Surg 2016;103:1487-96.

31. Yamamoto N, Hayashi K, Tanzawa Y, et al. Treatment strategies for well-differentiated liposarcomas and therapeutic outcomes. Anticancer Res 2012;32:1821-5.

32. Toulmonde M, Le Cesne A, Mendiboure J, et al. Longterm recurrence of soft tissue sarcomas: prognostic factors and implications for prolonged follow-up. Cancer 2014;120:3003-6.

33. Belal A, Kandil A, Allam A, et al. Malignant fibrous histiocytoma: a retrospective study of 109 cases. Am J Clin Oncol 2002;25:16-22.

34. Gibbs JF, Huang PP, Lee RJ, et al. Malignant fibrous histiocytoma: an institutional review. Cancer Invest 2001;19:23-7.

35. Peiper M, Zurakowski D, Knoefel WT, et al. Malignant fibrous histiocytoma of the extremities and trunk: an institutional review. Surgery 2004;135:59-66.

36. Yonemoto T, Tatezaki S, Ishii T, et al. Histological vascular invasion by tumors is a risk factor for distant metastasis in malignant fibrous histiocytoma. Anticancer Res 2005;25:1337-42.

37. Fiore M, Grosso F, Lo Vullo S, et al. Myxoid/round cell and pleomorphic liposarcomas: prognostic factors and survival in a series of patients treated at a single institution. Cancer 2007;109:2522-31.

38. Gebhard S, Coindre JM, Michels JJ, et al. Pleomorphic liposarcoma: clinicopathologic, immunohistochemical, and follow-up analysis of 63 cases: a study from the French Federation of Cancer Centers Sarcoma Group. Am J Surg Pathol 2002;26:601-16.

39. Ghadimi MP, Liu P, Peng T, et al. Pleomorphic liposarcoma: clinical observations and molecular variables. Cancer 2011;117:5359-69.

40. Hornick JL, Bosenberg MW, Mentzel T, et al. Pleomorphic liposarcoma: clinicopathologic analysis of 57 cases. Am J Surg Pathol 2004;28:1257-67.

41. Oliveira AM, Nascimento AG. Pleomorphic liposarcoma. Semin Diagn Pathol 2001;18:274-85.

42. Estourgie SH, Nielsen GP, Ott MJ. Metastatic patterns of extremity myxoid liposarcoma and their outcome. J Surg Oncol 2002;80:89-93.

43. Muratori F, Bettini L, Frenos F, et al. Myxoid Liposarcoma: Prognostic Factors and Metastatic Pattern in a Series of 148 Patients Treated at a Single Institution. Int J Surg Oncol 2018;2018:8928706.

44. Schwab JH, Boland P, Guo T, et al. Skeletal metastases in myxoid liposarcoma: an unusual pattern of distant spread. Ann Surg Oncol 2007;14:1507-14.

45. ten Heuvel SE, Hoekstra HJ, van Ginkel RJ, et al. Clinicopathologic prognostic factors in myxoid liposarcoma: a retrospective study of 49 patients with longterm follow-up. Ann Surg Oncol 2007;14:222-9.

46. Anghileri M, Miceli R, Fiore M, et al. Malignant peripheral nerve sheath tumors: prognostic factors and survival in a series of patients treated at a single institution. Cancer 2006;107:1065-74.

47. Zou C, Smith KD, Liu J, et al. Clinical, pathological, and molecular variables predictive of malignant peripheral nerve sheath tumor outcome. Ann Surg 2009;249:1014-22.

48. Wong WW, Hirose T, Scheithauer BW, et al. Malignant peripheral nerve sheath tumor: analysis of treatment outcome. Int J Radiat Oncol Biol Phys 1998;42:351-60.

49. Schaefer IM, Hornick JL, Barysauskas CM, et al. Histologic Appearance After Preoperative Radiation 
Therapy for Soft Tissue Sarcoma: Assessment of the European Organization for Research and Treatment of Cancer-Soft Tissue and Bone Sarcoma Group Response Score. Int J Radiat Oncol Biol Phys 2017;98:375-83.

50. Ghazala CG, Agni NR, Ragbir M, et al. Myxofibrosarcoma of the extremity and trunk: a multidisciplinary approach leads to good local rates of LOCAL control. Bone Joint J 2016;98-B:1682-8.

51. Haglund KE, Raut CP, Nascimento AF, et al. Recurrence patterns and survival for patients with intermediate- and high-grade myxofibrosarcoma. Int J Radiat Oncol Biol Phys 2012;82:361-7.

52. Roland CL, May CD, Watson KL, et al. Analysis of Clinical and Molecular Factors Impacting Oncologic Outcomes in Undifferentiated Pleomorphic Sarcoma. Ann Surg Oncol 2016;23:2220-8.

53. Gronchi A, Strauss DC, Miceli R, et al. Variability in Patterns of Recurrence After Resection of Primary Retroperitoneal Sarcoma (RPS): A Report on 1007 Patients From the Multi-institutional Collaborative RPS Working Group. Ann Surg 2016;263:1002-9.

54. Tseng WW, Pollock RE, Gronchi A. The Trans-Atlantic Retroperitoneal Sarcoma Working Group (TARPSWG): "Red wine or white"? Ann Surg Oncol 2016;23:4418-20.

55. Lahat G, Anaya DA, Wang X, et al. Resectable welldifferentiated versus dedifferentiated liposarcomas: two different diseases possibly requiring different treatment approaches. Ann Surg Oncol 2008;15:1585-93.

56. Gronchi A, Miceli R, Allard MA, et al. Personalizing the approach to retroperitoneal soft tissue sarcoma: histologyspecific patterns of failure and postrelapse outcome after primary extended resection. Ann Surg Oncol 2015;22:1447-54.

57. Singer S, Antonescu CR, Riedel E, et al. Histologic subtype and margin of resection predict pattern of recurrence and survival for retroperitoneal liposarcoma. Ann Surg 2003;238:358-70; discussion 370-1.

58. Tseng WW, Madewell JE, Wei W, et al. Locoregional disease patterns in well-differentiated and dedifferentiated retroperitoneal liposarcoma: implications for the extent of resection? Ann Surg Oncol 2014;21:2136-43.

59. Gholami S, Cassidy MR, Kirane A, et al. Size and Location are the Most Important Risk Factors for Malignant Behavior in Resected Solitary Fibrous Tumors. Ann Surg Oncol 2017;24:3865-71.

60. Beitler AL, Virgo KS, Johnson FE, et al. Current followup strategies after potentially curative resection of extremity sarcomas: results of a survey of the members of the society of surgical oncology. Cancer 2000;88:777-85.

61. Gerrand CH, Billingham LJ, Woll PJ, et al. Follow up after Primary Treatment of Soft Tissue Sarcoma: A Survey of Current Practice in the United Kingdom. Sarcoma 2007;2007:34128.

62. Sakata K, Johnson FE, Beitler AL, et al. Extremity soft tissue sarcoma patient follow-up: tumor grade and size affect surveillance strategies after potentially curative surgery. Int J Oncol 2003;22:1335-43.

63. Tzeng CW, Smith JK, Heslin MJ. Soft tissue sarcoma: preoperative and postoperative imaging for staging. Surg Oncol Clin N Am 2007;16:389-402.

64. Royce TJ, Punglia RS, Chen AB, et al. Cost-Effectiveness of Surveillance for Distant Recurrence in Extremity Soft Tissue Sarcoma. Ann Surg Oncol 2017;24:3264-70.

65. Cheney MD, Giraud C, Goldberg SI, et al. MRI surveillance following treatment of extremity soft tissue sarcoma. J Surg Oncol 2014;109:593-6.

66. Patel SA, Royce TJ, Barysauskas CM, et al. Surveillance Imaging Patterns and Outcomes Following Radiation Therapy and Radical Resection for Localized Extremity and Trunk Soft Tissue Sarcoma. Ann Surg Oncol 2017;24:1588-95.

67. Messiou C, Moskovic E, Vanel D, et al. Primary retroperitoneal soft tissue sarcoma: Imaging appearances, pitfalls and diagnostic algorithm. Eur J Surg Oncol 2017;43:1191-8.

68. Podoloff DA, Ball DW, Ben-Josef E, et al. NCCN task force: clinical utility of PET in a variety of tumor types. J Natl Compr Canc Netw 2009;7 Suppl 2:S1-26.
Cite this article as: Zaidi MY, Cardona K. Post-operative surveillance in soft tissue sarcoma: using tumor-specific recurrence patterns to direct approach. Chin Clin Oncol 2018;7(4):45. doi: 10.21037/cco.2018.08.03 